\title{
UN MODELO DE REPRESENTACIÓN DE COMPLEJIDAD ARGUMENTATIVA: EL DISCURSO DE FRIEDMAN
}

\author{
Cristián Juan Noemi Padilla* \\ Universidad de La Serena \\ La Serena, Chile
}

\begin{abstract}
Resumen: A partir de un método cualitativo que sigue los procedimientos establecidos por la grounded theory, el trabajo propone un modelo de representación de la complejidad argumentativa, a nivel de discurso. Con el propósito de establecer la adecuación del mismo, el trabajo describe las estrategias formales de la organización macroestructural de la Introducción al texto Libertad de Elegir, de Milton Friedman, con atención sobre la categoría de 'topos', por estimar que constituye un recurso formal a través del cual se despliega discursivamente la ideología en la textura, densidad o complejidad discursiva. El análisis ha puesto en evidencia una organización discursiva compleja que permite soportar las conclusiones del autor, en un marco ideológico que las avala y sustenta.
\end{abstract}

Palabras clave: Complejidad argumentativa. Textura discursiva. Topos. Macroestructura.

1 PROPÓSITO

El trabajo propone un modelo de representación de complejidad argumentativa a nivel de discurso, el que procura a su vez establecer parte de las estrategias textuales formales de nivel macroestructural que provocan densidad, textura o entramado discursivo y que, según se postula, están orientadas a contribuir a la coherencia argumental.

La noción de complejidad argumentativa ha sido abordada muy parcialmente desde diversas perspectivas, tales como la 'teoría de discusión razonable' (VAN EEMEREN; GROOTENDORST, 2004); la 'teoría de resolución de conflictos' (DONOHUE; KOLT, 1992); o la 'teoría de persuasión' (PERLOFF, 1993). Igualmente se han hecho algunos intentos tendientes a cuantificar, por medio de métodos estadísticos, dicha complejidad (JOVICIC, 2003).

El modelo que se propone incorpora la noción retórica clásica de 'topos', sugiriendo, por una parte, que ésta actúa en el texto a modo de gozne entre el conjunto de proposiciones de la macroestructura $\mathrm{y}$, por otra, que permite develar el marco ideológico desde el cual la macroestructura se proyecta.

Con vistas a establecer el nivel de adecuación del modelo, en el trabajo se analiza la porción superestructural 'Introducción' del texto Libertad de Elegir, de Milton Friedman (1983), procurando develar formalmente la ideología implícita que sirve de sustento a su propuesta teórico-económica.

\footnotetext{
* Este trabajo se enmarca en el proyecto de investigación FONDECYT N 1130584.

${ }^{* *}$ Profesor Titular, Doctor en Filología. Email: cnoemi@userena.cl.
} 
Diversas observaciones sobre lenguas no emparentadas filogenéticamente han permitido comprobar que el lenguaje no designa una realidad preexistente a él, de forma que se admite que las lenguas no constituyen un medio para representar la 'realidad', sino más bien un mecanismo que organiza particularmente nuestro 'mundo'.

Así por ejemplo, mientras que a través de una lengua como el español nos es posible categorizar una pretendida acción de la 'realidad' mediante el concepto 'comer', la lengua aymara por el contrario nos faculta para distinguir en ese mismo espacio acciones distintas según sea si ésta es realizada por un adulto /manxam/, por un niño /oxotsasim/, si la acción se proyecta sobre un hueso /hatum/, si lo hace sobre maíz /thurum/, sobre carne /tsitsim/, harina /hacum/, crema /allpim/, o puré /papim/.

En otras palabras, se ha admitido que las categorizaciones lingüísticas no son clasificaciones que emanan 'naturalmente' desde la realidad, sino más bien categorizaciones impuestas al continuum de la realidad, por medio de la facultad que permite el lenguaje.

Por esta razón, pareciera ser que las lenguas no constituyen procedimientos neutros desde un punto de vista ideológico, sino precisamente lo contrario, mecanismos que establecen categorizaciones no previas a nuestra experiencia, teñidas por una visión particular y que sirven para configurar, por tanto, sólo 'interpretaciones' que contribuyen a conformar así el mundo para nosotros. En este sentido, Van Dijk, por ejemplo, caracteriza la ideología como "[...] la base de las representaciones sociales compartidas por los miembros de un grupo [...]" (2000, p. 21), representación que no es sino posible en y por medio del lenguaje.

Como consecuencia parcial de lo anterior, la práctica totalidad de las formas de discurso que circulan en uno u otro sentido en los circuitos culturales (a excepción quizás de un cierto número de discursos 'científicos') no refieren necesariamente una 'verdad' en sentido cartesiano, sino más bien un conjunto de 'verdades culturales' sustentadas argumentativamente en la noción de 'verosimilitud', que surge a partir del contraste de los diferentes pareceres con opción a confrontamiento.

Al elaborar discurso, en efecto, no solamente se procura construir una representación de un determinado 'mundo' posible o de unos determinados 'hechos' con vistas a que sean simplemente compartidos por un destinatario; junto a ello, se intenta que los actos por medio de los cuales se refieren estos 'hechos' sean convenientemente juzgados por los receptores como 'adecuados' y que, las opiniones relativas al 'mundo' o a los 'hechos' expresadas en el contenido proposicional del enunciado sean consideradas, a su vez, como verosímiles.

\subsection{LA ESTRUCTURA ARGUMENTATIVA}

Toda práctica discursiva constante tiende a fijar socialmente una cierta 'estructura argumentativa', la cual es expresión de una proyección de los diversos discursos dominantes en uso sobre el sistema social: una estructura siempre 'ausente-presente', de 
fuerte profundidad psicosocial en la conciencia colectiva, pautada tanto por patrones cognitivos como culturales (en la forma de 'verdades culturales' socialmente admitidas).

Tal 'estructura argumentativa' se actualiza históricamente en la forma de sucesivos nuevos discursos (conversacionales, mediáticos, políticos, religiosos, educativos, etc.) que, de esta manera, la sancionan y reproyectan progresivamente en el tiempo y espacio, constituyendo una suerte de filtro en relación a lo que es posible 'decir' social y culturalmente (FOUCAULT, 1987).

Convengamos en que si el discurso no refiere directamente lo 'real', éste es más bien una construcción social de la realidad que se fija colectivamente para efecto de asegurar su continuidad histórica en la 'estructura argumentativa'. La estructura argumentativa del discurso se caracteriza parcialmente por sustentar valores no controversiales a través del empleo discursivo de ciertos 'topoi', patrones o moldes generales de razonamiento de base cultural, que sirven como premisas generales en el andamiaje discursivo argumentativo que, como tal, busca una predisposición de la conducta.

\subsection{IDEOLOGÍA}

El concepto de ideología ha sido tradicionalmente uno de los más complejos al interior de las ciencias sociales y además, uno de aquellos que se niega a desaparecer, aunque se haya intentado anunciar oficialmente su muerte más de una vez (FUKUYAMA, 1992). Por lo general se admite (LARRAÍN, 2008) que puede encerrar dos sentidos claramente diferenciados entre sí; un sentido negativo o crítico de la ideología; y otro más bien neutro o positivo.

La tradición marxista encierra ambos matices que en el propio Marx (1989) hay una oscilación en la cual el sentido crítico que conlleva se desplaza hacia uno más neutro de la misma. Autores como Althusser (1988) se inclinan más bien por el primer sentido, mientras que otros como Gramsci (1971) se muestran proclives más bien por la segunda opción. Dentro de la tradición marxista, el dilema fue considerar a la ideología como la expresión mistificada de los intereses de una clase en abierta contraposición con las otras clases (sentido crítico), o bien, al revés, hacer de la ideología el común denominador de la cultura (sentido neutro) de modo tal que cualquier manifestación de esta misma sería ideológica. La paradoja que se presenta entonces, tal como lo hace notar Manheim (1993), es que si toda expresión cultural (religiosa, filosófica, artística, política y hasta científica) resulta incapaz de escapar del estigma de ser tenida por 'ideológica', la teoría que trata de dar cuenta del concepto de ideología también sería ideológica padeciendo de idéntica ceguera que aquella otra que intenta denunciar.

Otros enfoques teóricos tienden por lo general a inclinarse por la segunda alternativa viendo en la ideología una función social que asegura la pertenencia a un grupo o a una cultura en particular (GEERTZ, 1988). En nuestro enfoque, nos inclinamos por una solución de carácter alternativo (RICOEUR, 1989) reconociendo en la ideología una función primordial que consiste en asegurar la articulación simbólica de 
la sociedad en sus variadas formas (política, religiosa, etc.) la cual puede convertirse en la expresión deformadora de los intereses de una clase como lo pretende la tradición marxista; o bien, puede actuar también como una instancia de legitimación (WEBER, 2007) permitiendo cubrir el vacío que se genera entre la pretensión de las autoridades políticas de que su autoridad sea considerada legítima por parte de la ciudadanía y la percepción que ésta última tiene de la mayor o menor legitimidad que le asiste a la autoridad para exigir semejante reconocimiento.

En este sentido la ideología no sólo sirve para deformar sino que además permite asegurar un grado de legitimidad en el ejercicio del poder, garantizando por añadidura el reconocimiento de los ciudadanos a este ejercicio. Ricoeur (1989) plantea inclusive lo que él llama la producción de una plusvalía, responsabilidad de la ideología en cuanto encargada por una parte de cubrir el vacío que se genera entre la pretensión de la autoridad y la adhesión de la ciudadanía; y por otra, de producir un excedente, vale decir, otorgarle al ejercicio del poder más de lo que la propia autoridad demanda.

De acuerdo a Ricoeur (1989), la ideología cumple tres funciones. En primer lugar, una función deformadora que sirve para encubrir los intereses de clase. Luego, una función legitimadora que asegura mediante la producción de una plusvalía que el ejercicio del poder por parte de la autoridad sea considerado como legítimo a ojos de la ciudadanía; por último, una función cohesionadora que sirve para asegurar la pertenencia a un grupo y su sentido de identidad.

Estas tres funciones se derivan de una función mucho más básica y primaria, a la que Ricoeur da el nombre de función representativa y que consiste en la producción simbólica (a través de la representación religiosa, filosófica, artística, etc.) por medio de la cual una sociedad se asegura a sí misma una identidad, solventando la necesidad de una necesaria legitimidad en el ejercicio del poder. Sin dicha función la ideología no podría cumplir ninguna de las consecuentes.

En el marco de este trabajo admitimos que la deformación, la legitimación y hasta la cohesión e identificación culturales descansan en la función representativa que cumple la ideología y que le asegura larga pervivencia como concepto al interior de las ciencias sociales.

\section{TOPOS}

A partir de Aristóteles (1990), y en el corpus doctrinario de la práctica totalidad de la teoría retórica clásica, se encuentra una categoría poco tratada a la que se le conoce originalmente con el nombre de tópica. En opinión del estagirita, a través de los silogismos dialécticos y retóricos se 'dicen' los topoi, lo que en opinión de Billig (1988) agregan una cualidad moral a nuestros actos de habla, pues refieren acuerdos de sentido socialmente sancionados.

Aristóteles (1990) distingue entre una tópica mayor y una tópica menor. La tópica mayor atraviesa cualquier discurso en forma transversal, independientemente de su asunto. Así, por ejemplo, algunos topoi como 'el más y el menos', 'lo posible y lo imposible', 'lo que ocurrió o no ocurrió', son transversales a todo tipo de discurso, sin 
importar su tema, aunque se advierta cierta relación entre topoi y género textual. El topos del 'más y el menos', en efecto, parece más propio para el género epidíctico, mientras que el de 'lo posible o imposible' más adecuado al género deliberativo. De otra parte, la tópica menor contiene todos aquellos topoi que serían adecuados en una ciencia en particular.

En una línea epistémica similar, en el enfoque de Perelman y Olbrechts-Tyteca (1989) se propone a este respecto que cuando se pretende fundamentar discursivamente valores o reforzar la intensidad de la adhesión que suscitan, los hablantes pueden recurrir a premisas de carácter general, a las que, siguiendo la tradición ortodoxa, denominan igualmente como topoi.

Ducrot (1986), al estudiar el potencial argumentativo de la lengua, propone diferenciar dos tipos de topoi, los extrínsecos y los intrínsecos. Los primeros son aquellos que, en su opinión, guardan relación con la estructura misma de la lengua, y por tanto se manifiestan en palabras gramaticales (relacionales). Los extrínsecos, de otra parte, más próximos a la concepción clásica del concepto, son aquellos que sirven de garante al encadenamiento entre dos enunciados al proporcionar un nexo con arreglo a una ética de lo posible, en función de un determinado contexto social. En su opinión, corresponden a principios ideológicos, compartidos por una comunidad lingüística más o menos extensa, y que si bien sirven para la construcción arbitraria de representaciones ideológicas, se presentan como si fueran exteriores al locutor. El enfoque teórico de Ducrot, en consecuencia, descansa sobre el supuesto de que hay secuencias discursivas cuya articulación no puede ser prevista sobre la sola base de los así llamados contenidos informativos.

Por otra parte, Rigotti (2006) proporciona un enfoque mayormente integrado de la noción de topos en el marco de una teoría parcial del lenguaje. En su opinión, el topos constituye una parte del 'módulo de estrategia argumentativa' y cumple una función de elemento generador del enunciado, en cuanto unidad de la dimensión institucionalizada del contexto.

Nuestro interés se ha focalizado en describir el rol que cumple la categoría de 'topos' en la dinámica del entramado, textura o complejidad del discurso. Desde nuestra perspectiva, el topos cumple la función de proporcionar apoyo o soporte, lo que aquí denominamos 'anclaje', en el entramado argumental de cada macroestructura.

El enfoque utilizado ha permitido considerar a los topoi como un elemento más del sistema de la lengua que forma parte de la competencia de los hablantes naturales, y que por tanto, es internalizado y compartido a través de procesos de socialización, concebida como adquisición de la gramática de los juegos del lenguaje.

En efecto, al aprender una lengua no solo se aprende un repertorio de normas gramaticales, sino que en aquella 'adquisición de determinadas reglas de juego de lenguaje' se interioriza, también, un determinado sistema de valores que establece representaciones particulares de la realidad.

De acuerdo con nuestro parecer, el topos forma parte de la expresión del sistema de valores de una comunidad. Colabora a la textura, densidad o complejidad del discurso para de este modo contribuir a la intencionalidad del sentido con arreglo a un 
contexto determinado, y consecuentemente constituye una marca de coherencia discursiva.

Los topoi están en la base de toda argumentación porque objetivan el sistema de valores. Los topoi son la marca de la presencia de valores en el discurso cuya función como elemento aglutinante se reconoce como evidencia de coherencia textual, puesto que 'dan la razón', es decir: avalan, respaldan y, refuerzan; esto es, contribuyen a la coherencia semántica y a la verosimilitud del discurso.

\section{TÓPICA E IDEOLOGÍA}

A partir de Aristóteles (2006), y en dependencia del tipo de discurso a través del cual se manifiesten preferentemente, distinguimos tres ejes axiológicos: el eje cardinal, el teologal y el pragmático. La noción de 'eje axiológico' pretende referir el dominio fundamental de los tipos de valores que se plasman en un determinado discurso.

El concepto de valor se puede concebir desde dos perspectivas básicas: una subjetiva y otra más objetiva (FRONDIZI, 1972). Desde una óptica subjetiva, el valor es el resultado del juicio que una persona formula frente a una determinada circunstancia, en dependencia de una multiplicidad de factores, tales como los gustos personales, el grado de información sobre una determinada materia, etc. Desde una perspectiva objetiva, el valor es independiente del parecer del sujeto y refiere una suerte de cualidad inherente a ciertos 'objetos', tal como el valor estético que se le atribuye, por ejemplo, en una determinada cultura, a ciertas obras de artes.

El eje cardinal debe su nombre a las cuatro virtudes llamadas cardinales o también primarias: la prudencia, la fortaleza, la humildad y la tolerancia (ARISTÓTELES, 2006). El eje teologal toma su denominación de las virtudes llamadas del mismo nombre que incorpora el cristianismo: la fe, la esperanza y la caridad.

Por último, el eje pragmático debe su designación al llamado logos pragmático (ARISTÓTELES, 2000), que a diferencia del logos apofántico, más propio de la ciencia y que descansa en la primacía que adquiere la lógica proposicional, reposaría más bien en la acción. El logos pragmático es propio de la política, en oposición al logos apofántico, que es el logos propio de la ciencia, y al logos fantástico que sería más bien el tipo de logos que prevalecería en la poesía y en el arte (ARISTÓTELES, 2000).

\section{ANÁLISIS}

\subsection{METODOLOGÍA}

En el trabajo se analizó inductivamente la porción superestructural 'Introducción' del libro Libertad de Elegir de Milton Friedman (1983), la que fue digitalizada para configurar el documento primario con el fin de fragmentarlo y examinarlo por párrafos. El procedimiento permitió la generación de etiquetas verbales que posteriormente se categorizaron para construir un modelo descriptivo. Para este efecto, se empleó un método cualitativo de análisis de discurso, aplicándose los procedimientos de la 
grounded theory (STRAUSS; CORBIN, 1998), a través del software Atlas/ti, versión 5.0. De acuerdo con el modelo, el texto se sintetizó en categorías conceptuales que aparecieron contextualmente y a las cuales se les asignó un código abierto. Posteriormente, se procedió a agrupar los códigos según sus propiedades; de esta forma se pudo obtener un conjunto de códigos que conformó una unidad superior mediante el recurso de una codificación axial, obteniéndose como resultado un modelo compuesto por relaciones generales entre los elementos, lo que permitió generar una explicación teórica desde la base.

\subsection{ANCLAJE}

El complejo semiótico denominado discurso se conforma, al menos, por una serie de elementos verbales que se encuentran organizados y relacionados entre sí a través de vínculos de diversa naturaleza, algunos de índole más bien formal, como las correspondencias gramaticales, y otros de naturaleza menos 'visibles' como las diversas dependencias semánticas, por ejemplo.

La mayor o menor organización e interrelación de las partes del discurso contribuyen en parte a lo que se ha llamado la textura del discurso (CALSAMIGLIA, 2002), urdimbre, densidad o complejidad semiótica que en razón de su grado de formalización contribuye consecuentemente a la coherencia textual.

Se emplea aquí el término coherencia precisamente para hacer mención a la interrelacionalidad global (macroestructural) potencialmente existente en el texto (HALLIDAY; HASAN, 1976; SHAPIRO; HUDSON, 1991) la cual es dependiente de su estructura formal y de las relaciones que establecen las partes del mismo. En este sentido, cabe señalar que estas relaciones no son necesariamente lineales sino que se establecen en distintos niveles de 'profundidad' del discurso.

En el marco de este trabajo, concebimos la noción de anclaje como una de las posibilidades de conformación de la textura, densidad, o complejidad discursiva que se formaliza lingüísticamente a través del vínculo semántico que se establece entre una proposición (p) y el argumento (p2), función atribuible al topos, en tanto cumple una función de soporte de coherencia de la (p), (y consecuentemente, de manera parcial, de la macroestructura concebida en su totalidad).

A modo de ejemplo, el tipo de anclaje básico aparece representado en la Tabla 1., en donde se muestra una secuencia formada por dos proposiciones, la primera rotulada por el software en el corpus como '1:4', a través de la cual el enunciador sostiene, afirma o declara,

(1) 1:4 (p) "La pequeña corriente inicial aumentó tras la Revolución Norteamericana [...]"

y la segunda, rotulada como '1:5', que avala, soporta o respalda la precedente,

(2) $1: 5$ (p2) "[...]empujadas por la miseria y la tiranía y atraídas por un anhelo de libertad y prosperidad." 
articuladas entre sí por medio de un topos de nexo causal.

Tabla 1 - Anclaje básico

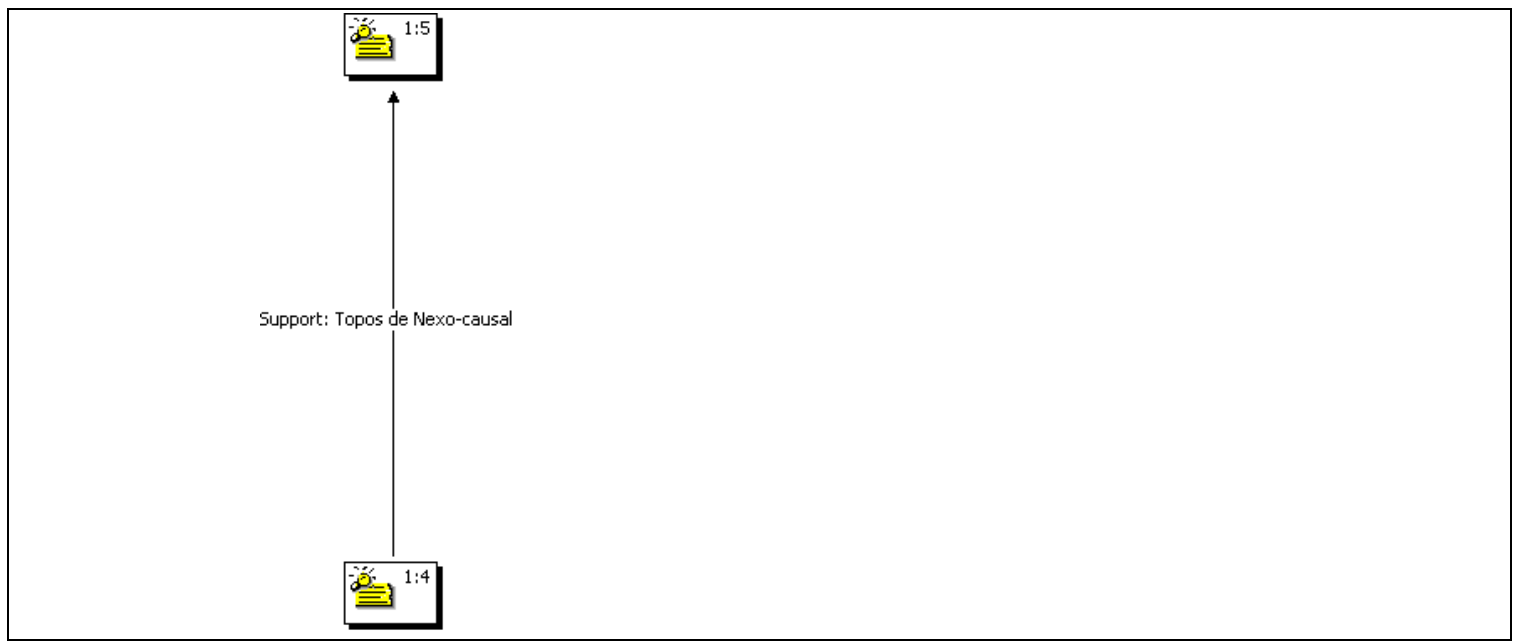

Cabe hacer notar que las proposiciones '1:4' y '1:5' se encuentran discursivamente vinculadas semánticamente por mediación de un topos, en este caso de tipo 'nexo causal', rotulado por el software en el corpus como 'support'. Hemos preferido referir el hecho como una vinculación discursiva para dar cuenta de la naturaleza contextual, social o pragmática del topos, que no obstante contribuye en cuanto elemento aglutinante, i.e., de anclaje, a la densidad, textura, complejidad y coherencia textual.

\subsection{RESULTADOS: LA COMPLEJIDAD ARGUMENTATIVA EN EL DISCURSO DE FRIEDMAN}

Como se ha dicho, en nuestra opinión, la noción de topos contribuye a la mayor o menor densidad (textura) de la macroestructura, y consecuentemente, a la mayor o menor coherencia semántica del discurso.

Como se muestra a partir de la Tabla 2., el análisis ha permitido develar lo que proponemos llamar la 'estructura argumentativa del discurso de Friedman', esto es textualización ideológica desplegada en tres niveles de textura, densidad o complejidad macroestructural argumental: soporte, conclusión y justificación, rotulados por el software en el corpus como support, explains y justifies, respectivamente, y que tiene por función articular discursivamente los ejes valóricos, a fin de conferir coherencia argumentativa global. 
Tabla 2 - Fragmento de la estructura argumentativa del discurso de Friedman

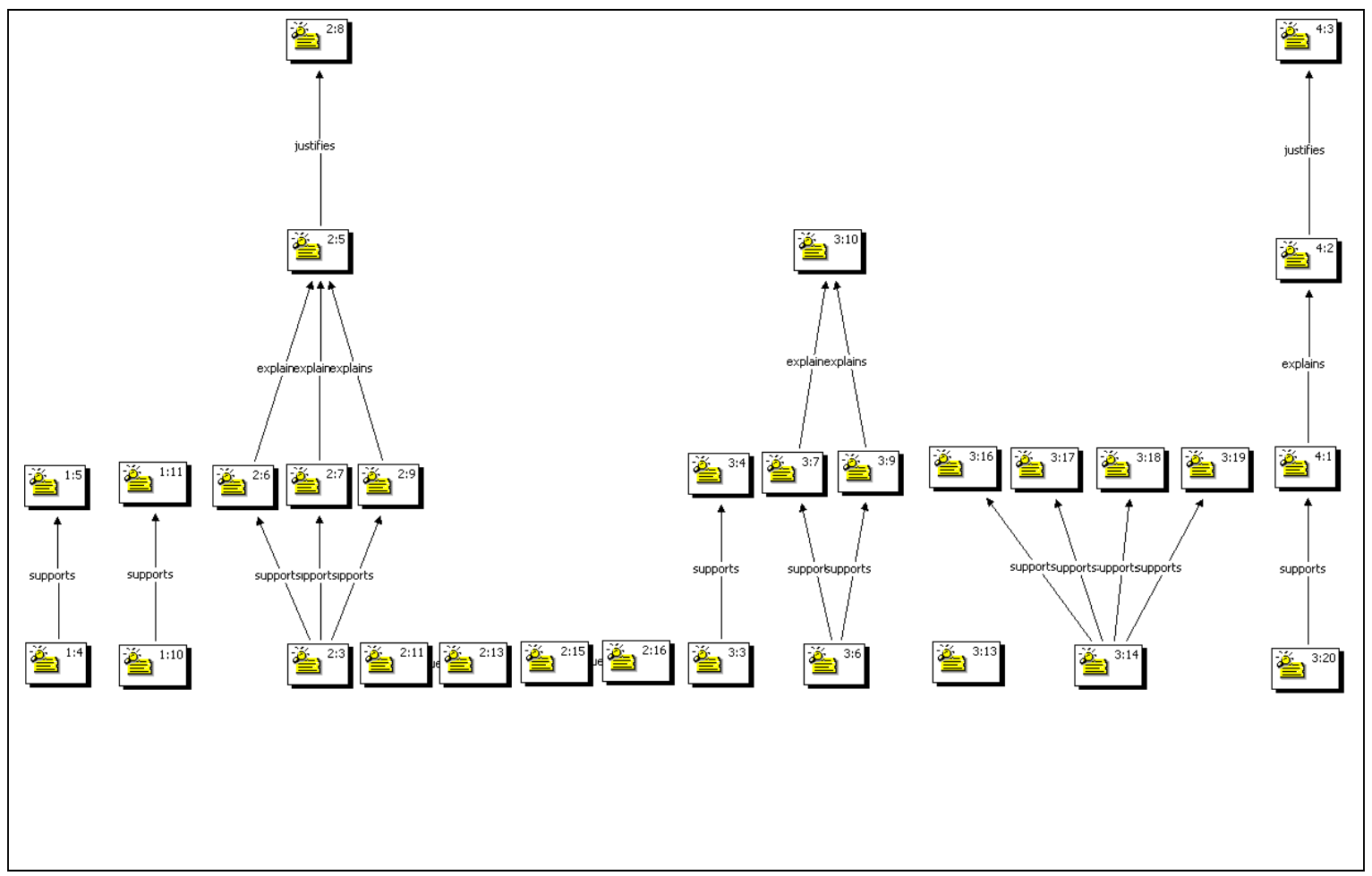

Como puede observarse, a pesar de que existen (p) desvinculadas de (p2), tal cual es el caso de '2:11'

(3) "Proclamó una nueva nación, la primera en la historia regida por el principio de que cada persona tenía derecho a perseguir sus propios intereses:"

o el de, '2:13',

(4) "Consideramos que estas verdades son evidentes por sí mismas, que todos los hombres han sido creados iguales, que su creador les ha dotado de ciertos derechos inalienables; que entre estos se encuentra la vida, la libertad y la búsqueda de la felicidad".

etc., la mayoría de ellas se encuentran ancladas, vinculadas o cohesionadas semánticamente con una (p2) como es el caso de '1:4' con '1:5' (cf. ejemplos (1) y (2) y Tabla 1),

'1:10' con '1:11'

(5)

1:10 (p) "La historia de Estados Unidos es la de un milagro económico y político hecho posible al ser llevados a la práctica dos grupos de ideas;" 
1:11 (p2) "[...] unas y otras, por una coincidencia curiosa, formuladas en documentos que se publicaron el mismo año de 1776."

vinculadas a través de un topos de nexo causal.

o bien, '3:3' con '3:4';

(6)

3:3 (p) "Gran parte de la historia de Estados Unidos gira alrededor del intento de poner en práctica los principios de la Declaración de la Independencia:"

3:4 (p2) "[...] desde la lucha por la abolición de la esclavitud resulta finalmente mediante una guerra civil cruenta, a la inspiración posterior a promover la igualdad de oportunidades, y al intento más reciente para conseguir la igualdad de resultados".

articuladas entre sí por un topos de ejemplo.

o, de otra parte, con más de una (p2), como por ejemplo la relación que establecen '2:3' con '2:6', '2:7' y '2:9'; o bien la que se conforma entre '3:6' con '3:7' y '3:9'.

2:3 (p) "El hallazgo clave de Adam Smith consistió en afirmar que todo intercambio voluntario genera beneficios para las dos partes y que, mientras la cooperación sea estrictamente voluntaria, ningún intercambio se llevará a cabo, a menos que ambas partes obtengan con ello un beneficio."

2:6 (p2) "No es necesaria una fuerza externa, la coerción o la violación de la libertad, para conseguir la cooperación entre individuos que se pueden beneficiar de ésta."·

vinculadas por un topos de nexo causal,

2:7 (p2) "Ni el hecho de que ese fin no formara parte de sus intenciones es siempre malo para la sociedad".

articuladas también por topos de nexo causal, y

2:9 (p2) "No he visto nunca que quienes dicen comerciar para el bien común hayan hecho mucho bien."

vinculadas por topos de incompatibilidad.

Resulta posible observar también la existencia de una (p) que establece tejido discursivo con cuatro (p2), como puede comprobarse en (8) donde '3:14'

3:14 (p) "Los Estados Unidos alcanzaron incluso una prosperidad mayor que la de Gran Bretaña, porque en América se comenzaba partiendo de cero: [...]" 
aparece vinculada con '3:16',

3:16 (p2) "[...] menos vestigios de clase y de status; $[\ldots] "$

por medio de un topos de nexo causal; de la misma forma con '3:17'

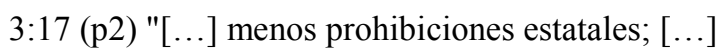

anclada igualmente por topos de nexo causal; igualmente con '3:18'

3:18 (p2) [...] un terreno más fértil para la energía, el empuje y la innovación $[\ldots]$

relacionada también por topos de nexo causal; y finalmente con '3:19'

3:19 (p2) "Y un continente vacío para conquistar."

anclada igualmente por topos de nexo causal.

Es posible observar igualmente que casi la mitad de las (p2) del discurso se encuentran vinculadas al segundo nivel de textura discursiva, i.e., la 'conclusión', relación semántica que patentiza el software, por ejemplo, mediante la vinculación que se establece entre las (p2) '2:6', '2:7' y '2:9' con la (p3) '2:5'.

(9)

2:6 (p2) "No es necesaria una fuerza externa, la coerción, o la violación de la libertad para conseguir la cooperación entre individuos que se pueden beneficiar de ésta."

2:7 (p2) "Ni el hecho de que ese fin no formara parte de sus intenciones es siempre malo para la sociedad."

2:9 (p2) "No hemos visto nunca que quienes dicen comerciar para el bien común hayan hecho mucho bien."

entramadas con la (p3) '2:5'

2:5 (p3) "Tal es la razón por la que, como dice Adam Smith, un individuo que 'intenta solamente su propio beneficio' es 'conducido por una mano invisible a alcanzar un fin que no formaba parte de sus intenciones."

en un eje valórico de naturaleza pragmática.

El mismo tipo de entramado discursivo se establece entre la (p2) '3:7' y '3:9' con la $\mathrm{p}(3)^{\prime} 3: 10$ ' 
3:7 (p2) "Al permitir que las personas cooperen entre sí sin la coacción de un centro decisorio, la libertad económica reduce el área sobre la que se ejerce el poder político."

3:9 (p2) "Además al descentralizar el poder económico, el sistema de mercado compensa cualquier concentración de poder político que pudiera producirse."

vinculadas con ' $3: 10$ '

3:10 (p3) "La combinación de poder político y económico en las mismas manos es una fórmula segura para llegar a la tiranía"

en una orientación axiológica igualmente pragmática.

En un sentido similar, la (p2) ' $4: 1$ '

4:1 (p2) "Cuando se produjo la Declaración de la Independencia, menos de tres millones de personas de procedencia europea y africana (es decir, excluyendo los indios nativos) ocupaban una estrecha franja a lo largo de la costa este de los Estados Unidos"

se entrama discursivamente con la (p3) '4:2'

4:2 (p3) "La agricultura era la actividad económica principal. De cada veinte trabajadores, diecinueve eran necesarios para alimentar al país y conseguir un excedente para exportarlo a cambio de productos extranjeros."

en un eje valórico igualmente pragmático.

Como observación colateral de la relación existente entre forma y sentido cabe advertir los lugares superestructurales donde se alcanza este nivel de densidad, textura o complejidad argumental en el discurso de Friedman: a partir de la (p) '2:3', de la (p)'3:6', y de la (p) '3:20', lo que aparentemente contribuye también a mantener la 'tensión' conclusiva y consecuente coherencia discursiva.

El discurso de Friedman alcanza en dos ocasiones el máximo nivel de textura, densidad o complejidad argumental, la 'justificación', a través de la vinculación semántica que se establece entre la (p3) '2:5' y la (p4) '2:8';

2:5 (p3) "Tal es la razón por la que, como dice Adam Smith, un individuo que 'intenta solamente su propio beneficio' es 'conducido por una mano invisible a alcanzar un fin que no formaba parte de sus intenciones." 
relacionada con

2:8 (p4) "Al perseguir sus propios intereses, el individuo promueve a menudo los de la sociedad de un modo más efectivo que cuando intenta directamente promoverlos".

concluyendo el entramado discursivo en un marco axiológico pragmático en el cual encuentra su sentido.

Igualmente la que se conforma mediante la relación que contraen la (p3) '4:2' y la (p4) ' $4: 3$ '.

4:2 (p3) "La agricultura era la actividad económica principal. De cada veinte trabajadores, diecinueve eran necesarios para alimentar al país y conseguir un excedente para exportarlo a cambio de productos extranjeros."

vinculada con

4:3 (p4) "Hoy día se necesita menos de uno de cada veinte trabajadores para alimentar a los 220 millones de norteamericanos y conseguir un excedente que convierte a los Estados Unidos en el mayor país exportador de alimentos del mundo".

finalizando igualmente la trama discursiva en un eje valórico pragmático a través del cual se semantiza.

La densidad, textura o complejidad del discurso de Friedman, el que, como ya se ha mostrado, se despliega en tres niveles de complejidad macroestructural, junto con los vínculos que se establecen entre las respectivas (p) (p2) (p3) y (p4), en distintos grados de complejidad, hacen suponer un producto de alta coherencia semántico pragmática, tanto en virtud de la complejidad y urdimbre propiamente estructural, como del empleo de los topoi que permiten completar la justificación con arreglo a valores de la cultura.

Es significativo también el hecho de que el máximo nivel de complejidad, densidad o textura macroestructural se alcance a partir del gozne que permite el topos que se gatilla a partir de la (p) '2:3' y '3:20', por la posición superestructural de privilegio que ocupa la 'justificación' en el discurso.

\section{CONCLUSIONES}

En este trabajo se ha podido develar parcialmente tanto la ideología subyacente al discurso introductorio del texto Libertad de Elegir de Milton Friedman (1983), como también parte de las estrategias formales de la macroestructura que emplea, desde la cual proyecta aspectos de su visión de mundo.

El abundante número de (p2), y la densidad discursiva, en términos de anclajes, textura, complejidad y profundidad macroestructural, (p3) y (p4), pone en evidencia una 
notoria conciencia lingüística del autor orientada a configurar una visión de mundo, dirigida a propósitos en apariencia intencionalmente establecidos.

El trabajo permitió aislar un número determinado de topoi los que, como se ha propuesto, cumplen función formal de elemento aglutinador entre niveles constitutivos de la macroestructura, a modo de gozne, contribuyendo a la densidad, textura, y complejidad argumentativa, y consecuentemente, a la coherencia semántica.

En el discurso de Friedman se ha podido advertir el empleo estratégico de generación discursiva de cuatro topoi de autoridad, tres de dirección, dos de evidencia anecdótica, ocho de identidad, cuatro de incompatibilidad, veinte y uno de nexo causal, y ocho de ejemplo (PERELMAN; OLBRECHTS-TYTECA, 1989).

Los topoi que han sido aislados en el discurso de Friedman se vinculan claramente con una determinada parte del sistema de valores de una comunidad concreta, particularmente con aquellos de índole pragmática, por lo que trasuntan una particular visión de mundo del sujeto enunciador que le sirven como 'fondo' discursivo

Por la amplia utilización de topos de identidad y de nexo causal, parece resultar evidente que el discurso de Friedman se apoya sobre el eje axiológico pragmático. Esto parece consecuente con el marco naturalista en el que se mueve su propuesta políticoeconómica caracterizada por la creencia en la existencia de un 'orden natural' - el mercado - cuyo normal funcionamiento no debe verse interferido por los gobiernos bajo ninguna circunstancia.

Cabría entender este naturalismo en que se sustenta el discurso en cuestión como una concepción filosófica que sostiene tanto la anterioridad como también la prioridad desde un punto de vista ontológico y jurídico, de los individuos y de su derecho a la propiedad, frente al Estado. La libertad de los individuos para disponer de su propiedad y de su vida es absoluta y la única limitación que se le reconoce es el derecho de los otros individuos para hacer lo mismo.

Resulta particularmente visible, en este sentido, que los topoi que utiliza Friedman aluden a un orden 'natural' de las cosas, a partir del cual se proyecta a lo largo de su discurso, por medio del entramado, densidad o complejidad argumentativa, el eje de valor general pragmático. Es decir, el fundamento ideológico del enunciador se articula lingüísticamente sobre una lógica de la causalidad, propia de un determinado modelo ideológico-económico.

La densidad, textura o complejidad formal de la macroestructura, por su parte, garantiza tanto la fuerza ilocucionaria del discurso como su coherencia argumental y potencial juicio de adecuación de verosimilitud, por parte del receptor lector.

REFERENCIAS

ALTHUSSER, L. Filosofia y marxismo. Madrid: Siglo XX, 1988.

ARISTÓTELES. Retórica. Madrid: Centro de Estudios Constitucionales, 1990.

. Acerca de la interpretación. Madrid: Gredos, 2000.

Ética a Nicómaco. Buenos Aires: Gradifco, 2006. 
BILLIG, M. Common-places of the British Royal Family: a rhetorical analysis of plain and argumentative sense. Text, Amsterdam, v. 8, n.3, p. 191-217, 1988.

CALSAMIGLIA, H.; TUSÓN, A. Las cosas del decir: manual de análisis del discurso. Barcelona: Ariel, 2002.

DONOHUE, W.; KOLT, R. Managing interpersonal conflict. London: Sage Publications, 1992.

DUCROT, O. El decir y lo dicho: polifonía de la enunciación. Barcelona: Ediciones Paidós, 1986.

FOUCAULT, M. El orden del discurso. Barcelona: Tusquets Editores, 1987.

FRIEDMAN, M. Libertad de elegir. Buenos Aires: Ediciones Orbis S.A., 1983.

FRONDIZI, R. ¿Qué son los valores?: una introducción a la axiología. México D.F.: Fondo de Cultura Económica, 1972.

FUKUYAMA, F. El fin de la historia y el último hombre. Barcelona: Planeta, 1992.

GEERTZ, C. Interpretación de las culturas. Barcelona: Gedisa, 1988

GRAMSCI, J. El materialismo histórico y la filosofía de Benedeto Croce. Buenos Aires: Nueva Visión, 1971.

HALLIDAY, M.; HASAN, R. Cohesion in English. London: Longman, 1976.

JOVICIC, T. Evaluation of argumentative strategies. In: EEMEREN, F.; BLAIR J.; WILLARD, C.; SNOECK. H. (Eds.), PROCEEDINGS OF THE FIFTH CONFERENCE OF THE INTERNATIONAL SOCIETY FOR THE STUDY OF ARGUMENTATION. Amsterdam, 2003, p. 571-580.

LARRAÍN, J. El concepto de ideología. Santiago de Chile: Lom Ediciones, 2008.

MANHEIM, K. Ideología y utopía. México D. F.: Fondo de Cultura Económica, 1993.

MARX, K. Contribución a la crítica de la economía política. Moscú: Progreso, 1989.

PERELMAN, Ch.; OLBRECHTS-TYTECA, L. Tratado de la argumentación: la nueva retórica. Madrid: Gredos, 1989.

PERLOFF, R. The Dynamics of Persuasion. New Jersey: Lawrence Erlbaum Associates Publishers, 1993.

RICOEUR, P. Ideología y utopía. Barcelona: Gedisa, 1989.

RIGOTTI, E. Relevance of Context-bound loci to Topical Potential in the Argumentation Stage. Argumentation, v. 20, issue 4, p. 519-540, 2006.

SHAPIRO, L.; HUDSON, J. Children's scripts, stories, and personal narratives. In: MC CABE, A.; PETERSON, C. (Eds.), Developing narrative structure. Hillsdale, NJ: Lawrence Erlbaum Associates, 1991, p. 89-136.

STRAUSS, A.; CORBIN, J. Basics of qualitative research - Techniques and procedures for developing grounded theory. London: Ed. Sage, 1998.

VAN DIJK, T. Ideología. Barcelona: Gedisa, 2000.

VAN EEMEREN, F.; GROOTENDORST, R. A systematic theory of argumentation. The Pragmadialectical Approach. Cambridge: Cambridge University Press, 2004.

WEBER, M. Ética protestante. Buenos Aires: Gradifco S. A., 2007.

Recebido em: 02/08/13. Aprovado em: 19/06/14

Title: A representation model of argumentative complexity: the Friedman speech

Author: Cristián Juan Noemi Padilla

Abstract: From a qualitative method following the procedures established by the grounded theory, this paper proposes a model for representing argumentative complexity at the discoursal level. In order to establish its suitability, this work describes the formal strategies in the macrostructural organization that are found in the introduction to Milton Friedman's Free to Choose. Special attention has been paid to the category of 'topos' since it constitutes a formal device through which ideology unfolds in discourse texture, density or complexity. The analysis revealed a complex discoursal organization that allows upholding the conclusions of the author within an ideological framework that sustains and supports them.

Keywords: Argumentative complexity. Discursive texture. Topos. Macrostructure. 
Título: Um modelo de representação de complexidade argumentativa: o discurso de Friedman Autor: Cristián Juan Noemi Padilla

Resumo: A partir de um método qualitativo que segue os procedimentos estabelecidos pela grounded theory, o trabalho propõe um modelo de representação da complexidade argumentativa, em nível de discurso. Com o objetivo de estabelecer a adequação desse modelo, o trabalho descreve as estratégias formais da organização macroestrutural da Introdução ao texto Libertad de Elegir, de Milton Friedman, com atenção à categoria de 'topos', por considerar que constitui um recurso formal através do qual se desdobra discursivamente a ideologia na textura, densidade ou complexidade discursiva. A análise pôs em evidência uma organização discursiva complexa que permite dar suporte às conclusões do autor, em um quadro ideológico que as confirma e sustenta.

Palavras-chave: Complexidade argumentativa. Textura discursiva. Topos. Macroestrutura. 